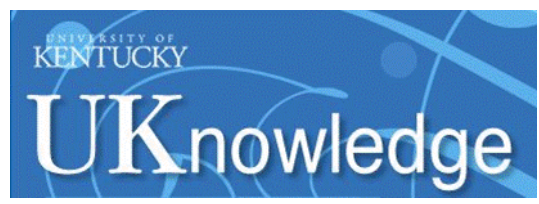

University of Kentucky

UKnowledge

$1-1-2017$

\title{
Neuropathological and Genetic Correlates of Survival and Dementia Onset in Synucleinopathies: A Retrospective Analysis
}

\author{
David J. Irwin \\ University of Pennsylvania \\ Murray Grossman \\ University of Pennsylvania \\ Daniel Weintraub \\ University of Pennsylvania \\ Howard I. Hurtig \\ University of Pennsylvania \\ John E. Duda \\ University of Pennsylvania \\ Follow this and additional works at: https://uknowledge.uky.edu/sbcoa_facpub
}

Part of the Diseases Commons, Neuroscience and Neurobiology Commons, and the Pathology See nextpage for additional authors

Right click to open a feedback form in a new tab to let us know how this document benefits you.

\section{Repository Citation}

Irwin, David J.; Grossman, Murray; Weintraub, Daniel; Hurtig, Howard I.; Duda, John E.; Xie, Sharon X.; Lee, Edward B.; Van Deerlin, Vivianna M.; Lopez, Oscar L.; Kofler, Julia K.; Nelson, Peter T.; Jicha, Gregory A.; Woltjer, Randy; Quinn, Joseph F.; Kaye, Jeffery; Leverenz, James B.; Tsuang, Debby; Longfellow, Katelan; Yearout, Dora; Kukull, Walter; Keene, C. Dirk; Montine, Thomas J.; Zabetian, Cyrus P.; and Trojanowski, John Q., "Neuropathological and Genetic Correlates of Survival and Dementia Onset in Synucleinopathies: A Retrospective Analysis" (2017). Sanders-Brown Center on Aging Faculty Publications. 128. https://uknowledge.uky.edu/sbcoa_facpub/128

This Article is brought to you for free and open access by the Aging at UKnowledge. It has been accepted for inclusion in Sanders-Brown Center on Aging Faculty Publications by an authorized administrator of UKnowledge. For more information, please contact UKnowledge@lsv.uky.edu. 


\title{
Neuropathological and Genetic Correlates of Survival and Dementia Onset in Synucleinopathies: A Retrospective Analysis
}

\author{
Digital Object Identifier (DOI) \\ https://doi.org/10.1016/S1474-4422(16)30291-5 \\ Notes/Citation Information \\ Published in The Lancet Neurology, v. 16, issue 1, p. 55-65. \\ (c) 2016 Elsevier Ltd. All rights reserved.
}

This manuscript version is made available under the CC-BY-NC-ND 4.0 license https://creativecommons.org/licenses/by-nc-nd/4.0/.

The document available for download is the author's post-peer-review final draft of the article.

\section{Authors}

David J. Irwin, Murray Grossman, Daniel Weintraub, Howard I. Hurtig, John E. Duda, Sharon X. Xie, Edward B. Lee, Vivianna M. Van Deerlin, Oscar L. Lopez, Julia K. Kofler, Peter T. Nelson, Gregory A. Jicha, Randy Woltjer, Joseph F. Quinn, Jeffery Kaye, James B. Leverenz, Debby Tsuang, Katelan Longfellow, Dora Yearout, Walter Kukull, C. Dirk Keene, Thomas J. Montine, Cyrus P. Zabetian, and John Q. Trojanowski 


\title{
Neuropathological and genetic correlates of survival and dementia onset in synucleinopathies: a retrospective analysis
}

\author{
David J. Irwin, MD, MSTR ${ }^{1,2}$, Murray Grossman, MD $^{1,2}$, Daniel Weintraub, MD ${ }^{1,2,4}$, Howard I. \\ Hurtig, MD ${ }^{1,2}$, John E. Duda, MD ${ }^{2,4}$, Sharon X. Xie, $\mathrm{PhD}^{3}$, Edward B. Lee, MD, PhD', \\ Vivianna M. Van Deerlin, MD, PhD ${ }^{1}$, Oscar L. Lopez, MD ${ }^{5}$, Julia K. Kofler, MD ${ }^{6}$, Peter T. \\ Nelson, MD, PhD ${ }^{7,8}$, Gregory A. Jicha, MD, PhD ${ }^{7}$, Randy Woltjer, MD, PhD ${ }^{9}$, Joseph F. \\ Quinn, MD ${ }^{10}$, Jeffery Kaye, MD ${ }^{10}$, James B Leverenz, MD ${ }^{13}$, Debby Tsuang, MD, MSc ${ }^{14,15}$, \\ Katelan Longfellow, MD ${ }^{11,16}$, Dora Yearout, BS ${ }^{11,15}$, Walter Kukull, PhD ${ }^{11}$, C. Dirk Keene, \\ MD, PhD ${ }^{12}$, Thomas J. Montine, MD, PhD ${ }^{11,12}$, Cyrus P. Zabetian, MD, MS ${ }^{11,15,16}$, and John \\ Q. Trojanowski, MD, PhD ${ }^{1}$
}

${ }^{1}$ Center for Neurodegenerative Disease Research, Department of Pathology and Laboratory Medicine, Morris K. Udall Parkinson's Disease Center Of Excellence, Institute on Aging, University of Pennsylvania Perelman School of Medicine, Philadelphia, PA 19104-6021, USA

${ }^{2}$ Department of Neurology, University of Pennsylvania Perelman School of Medicine, Philadelphia, PA 19104-6021, USA

${ }^{3}$ Department of Biostatistics and Epidemiology, University of Pennsylvania Perelman School of Medicine, Philadelphia, PA 19104-6021, USA

${ }^{4}$ Parkinson's Disease Research, Education and Clinical Center, Michael J. Crescenz VA Medical Center, Philadelphia, PA 19104, USA

${ }^{5}$ Department of Neurology, University of Pittsburgh, Pittsburgh, PA, USA

${ }^{6}$ Department of Pathology, University of Pittsburgh School of Medicine, Pittsburgh, Pennsylvania, USA

${ }^{7}$ Sanders-Brown Center on Aging, University of Kentucky, Lexington, KY, 40536, USA

${ }^{8}$ Department of Pathology, University of Kentucky, Lexington, KY, 40536, USA

${ }^{9}$ Department of Pathology, Oregon Health and Science University, Portland, OR, 97239, USA

${ }^{10}$ Department of Neurology, Oregon Health and Science University, Portland, OR, 97239, USA

${ }^{11}$ Department of Neurology, University of Washington School of Medicine, Seattle, WA 98195, USA

${ }^{12}$ Department of Pathology, and the Pacific Northwest Udall Center, University of Washington School of Medicine, Seattle, WA 98195, USA

Please send correspondence to: David J. Irwin, MD, Frontotemporal Degeneration Center/Center for Neurodegenerative Disease Research, University of Pennsylvania Perelman School of Medicine, Hospital of the University of Pennsylvania, 3600 Spruce Street, Philadelphia, PA 19104, (215)-662-3361, dirwin@mail.med.upenn.edu. 
${ }^{13}$ Cleveland Clinic Lou Ruvo Center for Brain Health, Cleveland Clinic Foundation, Cleveland, $\mathrm{OH}$ 44195, USA

${ }^{14}$ Department of Psychiatry and Behavioral Sciences University of Washington School of Medicine, Seattle, WA 98195, USA

${ }^{15}$ Geriatric Research, Education, and Clinical Center, VA Puget Sound Health Care System, Seattle, WA 98108

${ }^{16}$ Northwest Parkinson's Disease Research, Education and Clinical Center, VA Puget Sound Health Care System, Seattle, WA 98108

\section{Abstract}

Background-There exists great heterogeneity in patient survival and the time interval between motor symptom and dementia onset (MDI) across Lewy body spectrum disorders (LBSD). The goal of this study is to identify genetic and pathological findings that have the strongest association with these features of clinical heterogeneity in LBSD.

Methods-In this retrospective study, we examined symptom onset, and genetic and neuropathological data from a cohort of LBSD patients with autopsy-confirmed asynucleinopathy (as of Oct 1,2015) recruited from 5 clinical research centres in 5 cities in the USA. Using histopathology techniques and markers, we assessed the burden of tau neurofibrillary tangles, neuritic plaques, $\mathbf{a}$-synuclein inclusions, and other pathologic changes in cortical regions using averaged ordinal scores and genotyped cases for variants associated with LBSD. We evaluated the time interval from onset of motor symptoms to dementia (MDI) and overall survival in groups with varying levels of co-morbid Alzheimer's disease pathology (AD) according to current National Institute on Aging-Alzheimer's Association neuropathological criteria and used multivariate regression to control for age at death and gender.

Findings-This study included 213 patients who had been followed to autopsy and met inclusion criteria of clinical LBSD with autopsy-confirmed a-synculeinopathy. Patient groups were characterized by no $(n=49,23 \%)$, low-level $(n=56,26 \%)$, intermediate-level $(n=45,21 \%)$ or highlevel ( $\mathrm{n}=63,30 \%)$ AD neuropathology. Across groups of increasing levels of AD neuropathology, there were higher cerebral a-synuclein scores, shorter MDI, and shorter disease duration ( $\mathrm{p}<0.0001$ all). Multivariate regression found independent negative associations of cerebral tau score with MDI $(\beta=-4 \cdot 0,95 \% \mathrm{CI}-5.5$ to $-2 \cdot 6 ; \mathrm{p}<0.0001)\left(\mathrm{R}^{2}=0 \cdot 22, \mathrm{p}<0.0001\right)$ and with survival $(\beta=-2 \cdot 0,95 \% \mathrm{CI}-3 \cdot 2$ to $-0 \cdot 8 ; \mathrm{p}<0 \cdot 0001)\left(\mathrm{R}^{2}=0 \cdot 15, \mathrm{p}<0 \cdot 0001\right)$ in models including age at death, gender, cerebral neuritic plaque scores, cerebral a-synuclein, presence of cerebrovascular disease, $M A P T$ haplotype, and $A P O E$ genotype as covariates.

Interpretation-AD neuropathology is common in LBSD and confers a worse prognosis for each increasing level of neuropathological change. Cerebral neurofibrillary tau tangle burden, $a-$ synuclein pathology, and amyloid plaque pathology are the strongest pathological predictors of a shorter MDI and survival in LBSD. In the future, clinical diagnostic criteria which use reliable biomarkers for AD neuropathology in LBSD should help identify the most appropriate patients for clinical trials of emerging therapies targeting tau, amyloid-beta or a-synuclein, and stratify them by level of AD neuropathology. 
Funding-NIH (NIA/NINDS).

\section{Introduction}

Parkinson's disease dementia (PDD) ${ }^{1}$ and dementia with Lewy bodies (DLB) ${ }^{2}$ are considered to be on a spectrum of clinical manifestations of underlying Lewy body disease ${ }^{3}$ characterized by intra-neuronal inclusions composed of pathological a-synuclein (SYN) protein (i.e. synucleinopathies) ${ }^{4}$. The majority of patients with idiopathic Parkinson's disease (PD) will eventually develop dementia over the course of their illness ${ }^{5}$. However, the timing of the onset of dementia is highly variable, with some patients showing no signs of cognitive impairment for many years after the onset of $\mathrm{PD}^{6-8}$. In contrast, up to $25 \%$ of $d e$ novo $\mathrm{PD}$ patients have mild cognitive impairment (MCI), and incident $\mathrm{MCI}$ in patients with established PD can rapidly progress to PDD ${ }^{9}$. Furthermore, according to current consensus criteria $^{2}$, patients with DLB have dementia that precedes or occurs within one year after the onset of motor signs of parkinsonism. Although PDD and DLB are diagnostically categorized by the timing of symptom occurrence, the clinical features of cognitive and motor impairment are often indistinguishable, especially later in disease course ${ }^{1-3}$. While the underlying neuropathological and genetic influences on this variable expression of cognitive impairment across Lewy body spectrum disorders (LBSD) are currently unknown, we previously found that cortical SYN pathology was the strongest predictor of dementia in $\mathrm{PD}$ and $\mathrm{PDD}^{6}$. Moreover, patients with a clinical diagnosis of PDD who had significant Alzheimer's disease neuropathology (AD) had a shorter time interval from the onset of PD to the onset of dementia, and thus, more closely resembled the natural history of DLB ${ }^{6}$.

Here we test the hypothesis that co-morbid AD neuropathology (i.e. neuritic plaques, [NPs] and neurofibrillary tangles [NFTs]) associates with of the timing of the onset of dementia and of survival in LBSD.

\section{Methods}

\section{Participants}

Patients were recruited by local clinicians and study investigators as part of several preexisting clinical research program projects from clinical research centres associated with the Udall Center for Excellence in Parkinson's Disease Research at the University of Pennsylvania (Pennsylvania Parkinson's Disease \& Movement Disorders Center, Philadelphia VA Medical Center, Alzheimer's Disease Core Center, or Frontotemporal Degeneration Center), the Pacific Northwest Udall Center (University of Washington [UW], Seattle, WA, USA) and Oregon Health \& Science University ([OHSU] Portland, OR, USA), or clinical research centers associated with the Alzheimer's Disease Research Centers at UW, OHSU, the University of Pittsburgh, or Sanders-Brown Center on Aging at the University of Kentucky.

Patients who had been followed to autopsy at the corresponding institutional neuropathology lab as of October 1, 2015 were included if they met formal clinical criteria for either probable $\mathrm{DLB}^{2}$ or $\mathrm{PDD}^{10}$, as previously described, ${ }^{6}$ with autopsy confirmation of brainstem, transitional, or neocortical stage LBSD synucleinopathy ${ }^{2,4}$. One patient had a secondary 
neuropathological diagnosis of progressive supranuclear palsy tauopathy which confounds the examination of cortical AD tau pathology, and was thus excluded. A subset of neuropathological and genetic data presented here (from 103 individuals) were previously reported in a smaller cohort of patients in a different analysis of $\mathrm{PDD}^{6}$ or the frequency of genetic variants in LBSD ${ }^{11,12}$ compared with controls. All procedures were performed in accordance with local Institutional Review Board guidelines and approvals at each center, including written informed consent for autopsy and analysis of tissue sample data. Please see supplemental methods for further details on clinical data collection and referral centers.

\section{Procedures}

Neuropathological examination was performed (EBL, JQT, JBL, TJM, CDK, RW, JK, PTN) using standard methods and the same consensus diagnostic criteria at each center ${ }^{4,13}$. As part of this assessment, sections for each of 7 standardly sampled cortical/limbic regions were stained using accepted methods ${ }^{4,13}$ and graded on an $0-3$ ordinal scale ${ }^{4}$ for NFTs, cored or neuritic plaques, and SYN pathology. Please see supplemental methods for further details on the methods of staining at each center and other pathological variables. The average cerebral score for each pathological change was calculated as previously described ${ }^{6}$. Briefly, an average of the $0-3$ ordinal score ratings from these regions were used as a continuous measure of cortical pathology burden for NFTs, NPs and SYN (i.e. cerebral score). We used Braak neurofibrillary tangle stage and CERAD neuritic plaque score to classify AD neuropathology into four groups: no AD neuropathology; "low-level" AD; "intermediate-level" AD; and "high-level" AD using modified criteria (supplemental methods $)^{4,13}$. We also dichotomized the burden of AD neuropathology into SYN+AD (intermediate/high $\mathrm{AD}$ ) and SYN-AD (no/low AD) groups, as described previously ${ }^{6}$ to test the diagnostic accuracy of the MDI for detecting SYN+AD.

DNA was isolated from peripheral blood or frozen brain samples using standard techniques, ${ }^{6}$ and samples from individuals were genotyped for common single nucleotide polymorphisms (SNPs) in genes previously associated with $\mathrm{LBSD}^{11,14}$ —apolipoprotein $\mathrm{E}$ ( $A P O E \varepsilon \mathcal{\varepsilon} 2, \varepsilon 3$, and $\varepsilon 4$ via rs429358/rs7412), tau (MAPTH1 haplotype via risk allele "A" at rs1800547) and a-synuclein ( $S N C A$ risk allele "G" at rs356219) — by TaqMan assay (Life Technologies, Waltham, MA, USA). In addition, the entire glucocerebrosidase gene ( $G B A)$ coding region and all intron-exon boundaries were sequenced using PCR to detect known pathogenic mutations and the coding SNP p.E326K risk allele "G" at rs2230288 as described ${ }^{15}$. Missing data from cases with no or insufficient DNA samples were omitted from analysis. All genotyping was performed (CPZ) at the Pacific Northwest Udall Center (Seattle, WA, USA).

\section{Statistical Analyses}

Continuous variables were analyzed using parametric or non-parametric univariate tests as appropriate and categorical variables compared using a chi-square test analysis. Missing data was excluded from analyses. To test the diagnostic accuracy of using MDI cut-off of $\leq 1$ year $^{2}$ to distinguish DLB from PDD, we used receiver-operating characteristic (ROC) curve analyses for advanced SYN pathology (neocortical stage vs brainstem or limbic stages) or AD pathology (SYN+AD vs SYN-AD) patient groups. Correlations of cerebral NFT, NP, 
and SYN scores with clinical features (age at death, MDI, and survival) was performed using a Spearman correlation.

We used multivariate linear regression to test the independent associations of AD-related pathology (i.e. cerebral NFTs and NPs) with the dependent variables MDI and survival. Final models were derived from base models controlling for age at death and gender using Bayesian Information Criteria ${ }^{16}$. In the absence of an external validation sample, the final model was validated using a bootstrap procedure (i.e. internal validation) ${ }^{17}$. We report average beta estimates with $95 \%$ confidence intervals $(95 \% \mathrm{CI})$ from a bootstrapping random sampling procedure with 1,000 bootstrap samples. (Please see Supplementary methods for further details on model building procedure).

All analyses were 2-tailed ( $a=0.05)$. We adjusted correlation analyses to $a=0.003$ to correct for multiple comparisons and reduce the likelihood of false positive discovery, and performed using SPSS v23.0 (IBM, Chicago, IL, USA) or STATA v12.1 (StataCorp, College Station, TX, US).

\section{Role of the funding source}

The funder of the study had no role in study design, data collection/analysis/interpretation, or writing of the report. All authors had full access to all the data in the study and had final responsibility for the decision to submit for publication

\section{Results}

213 LBSD patients meeting clinical criteria for PDD or DLB who had been followed to autopsy by October 1, 2015 at 5 clinical research centres in 5 cities in the USA were selected for this study. Two patients were missing survival data and 27 patients were missing MDI data. Missing individual neuropathological and genetic variable data are listed in Tables 1-2.

Classification of LBSD cases based on stages of AD neuropathology ${ }^{4,13}$ revealed the following distribution (Table 1): 49 patients (23\%) had negligible levels of AD neuropathological change, i.e. No-AD); 56 patients (26\%) had low-level AD; 45 patients (21\%) had intermediate-level AD; and 63 (30\%) had high-level AD. As defined above, there were increasing stages of NFT and NP pathology across groups with increasing levels of AD neuropathology, including higher NFT/NP cerebral scores ( $<<0.0001$ both), CAA scores $(\mathrm{p}<0.0001)$, and NFT/NP scores in the basal ganglia (BG) $(\mathrm{p}<0.0001$, both). Evaluation of SYN pathology across increasing levels of $A D$ revealed a step-wise increase in a-synuclein stage $(\mathrm{p}=0 \cdot 018)$, cerebral SYN score $(\mathrm{p}<0 \cdot 0001)$, and BG SYN score $(\mathrm{p}=0.29)$. There were no significant differences between groups for the other neuropathological changes examined (Table 1).

Examination of genetic variants previously associated with LBSD ${ }^{11,12,14}$ (Table 2) revealed a decreasing frequency of heterozygous patients carrying the $G B A$ p.E326K risk allele or $G B A$ mutation with increasing levels of AD neuropathology (p=0.03, both). SNPs in MAPT and $S N C A$ were similar across groups. The frequency of the $A P O E \varepsilon 4$ allele was not 
associated with the four levels of increasing AD neuropathology, but in a dichotomous comparison of SYN+AD and SYN-AD groups finds patients with one or more copies of $A P O E \varepsilon 4$ were more frequent ( $\mathrm{p}=0.04)$ in the $\mathrm{SYN}+\mathrm{AD}$ group (Supplemental Table 1).

Examination of clinical demographics (Table 3) revealed an increasing number of patients with a clinical diagnosis of DLB (compared to PDD) with increasing levels of AD neuropathology $(\mathrm{p}<0 \cdot 0001)$ (Table 3). Despite the significantly higher frequency of DLB clinical phenotype in the SYN+AD group (Supplemental Table 1) there was no clear delineation of AD neuropathology groups according to clinical phenotype (Figure 1a). We used a ROC curve analysis to test diagnostic accuracy for SYN neocortical stage (Figure 1b; $\mathrm{AUC}=0.67$ ) and $\mathrm{SYN}+\mathrm{AD}$ (Figure 1c; $\mathrm{AUC}=0.72$ ) and found poor specificity for both. Thus, we did not find evidence of a SYN or AD neuropathological grouping to substantiate the MDI 1-year rule, which clinically distinguishes DLB from PDD according to current criteria $^{2}$.

Increasing level of $\mathrm{AD}$ neuropathology was associated with older age at motor onset $(\mathrm{p}<0 \cdot 0001)$, dementia onset $(\mathrm{p}=0.005)$, and death $(\mathrm{p}=0 \cdot 001)$, with significant contrasts between $\mathrm{AD}$ groups and the no-AD group (Table 3 ). The time interval from the onset of dementia to death was similar between groups. Increasing severity of AD was also associated with a stepwise decrement in MDI and survival (Figure 2) which was significant between intermediate and high-level AD groups compared with no-AD and low-level AD groups (Table 3). We also examined the individual association of Braak (NFT) and CERAD (NP) stages with MDI and survival, and found a similar step-wise association of increasing CERAD and Braak stages with shorter MDI and survival with more significant differences between individual Braak stages than CERAD stages (Figure 2, Supplemental Table 2).

We next examined the correlation of continuous measures (cerebral scores) of individual neuropathological changes with demographic features (Figure 3); the cerebral NFT, NP and SYN scores were all highly correlated with each other $(\mathrm{rho}=0 \cdot 4-0 \cdot 6, \mathrm{p}<0 \cdot 0001)$ and inversely correlated with the MDI (rho $=-0 \cdot 3--0 \cdot 4, \mathrm{p}<0 \cdot 0001)$ and survival (rho $=-0 \cdot 3-$ $-0 \cdot 4, \mathrm{p}<0.0001$ all). Cerebral pathology scores did not correlate with age at death.

Univariate linear regression models with MDI as the dependent variable found $A P O E$ genotype, and continuous measures of cerebral NFT, NP and SYN pathology all had significant associations (Supplemental Table 5). Our final multivariate model, controlling for age at death, gender and other neuropathological or genetic variables using optimal Bayesian Information Criteria found an inverse association with cerebral NFT score $(\beta=-4 \cdot 0,95 \%$ $\mathrm{CI}=-5 \cdot 8,-2 \cdot 6 ; \mathrm{p}<0 \cdot 0001)$ and a positive association of age at death $(\beta=0.2,95 \% \mathrm{CI}=0 \cdot 02$, 0.3; $\mathrm{p}=0.05)\left(\right.$ Model $\left.\mathrm{R}^{2}=0 \cdot 22, \mathrm{p}<0 \cdot 0001\right)($ Supplemental Table 5).

Univariate linear regression models with survival as the independent variable found $A P O E$ genotype $(\mathrm{p}=0.003)$ and continuous measures of cerebral NFT, NP and SYN pathology all had significant $(\mathrm{p}<0.0001)$ associations (Supplemental Table 6). Our final multivariate model found an independent negative association of cerebral NFT score $(\beta=-2 \cdot 04,95 \% \mathrm{CI}=$ $-3 \cdot 2,-0 \cdot 8, p=0 \cdot 003$ ) (Model $\left.R^{2}=0 \cdot 15, p<0 \cdot 0001\right)$ (Supplemental Table 6). Examination of interaction terms for cerebral NFT score with cerebral SYN score or with cerebral NP score 
and for $A P O E$ with cerebral NP or cerebral NFT were not significant and did not optimize Bayesian Information Criteria values of either model.

\section{Discussion}

We performed an analysis of the contribution of AD neuropathology to onset of dementia and to survival in LBSD through group-wise comparisons of four levels of AD neuropathology severity ${ }^{4}$. Our retrospective analysis in a large cohort of LBSD patients with detailed clinical, pathological, and genetic information found NFT accumulation to be the strongest correlate of a shorter MDI and overall survival. We found converging evidenceusing both a continuous measure of cerebral NFT burden score (Figure 3, Table 4) and sequential categorical Braak NFT stages (Figure 2, Supplemental Table 2)—which showed increasing levels of cerebral NFT burden with worsening of these signs of poor prognosis. Indeed, the high-level AD group had on average $\sim 7$ years shorter MDI and overall survival than the no-AD group (Table 3).

Other studies have also found shorter MDI and overall survival in autopsied PD patients with co-morbid AD neuropathology $8,18,19$ and a similar inverse association of cerebral NFT, NP, and SYN pathology with the MDI across LBSD. ${ }^{7}$ However, additional research has reported that $\mathrm{A} \beta$ plaques ${ }^{8,20}$ or a summation of NFT, A $\beta$, and SYN pathological changes ${ }^{21}$ were the strongest correlates of a shorter MDI in LBSD. There are several potential reasons for these discrepancies. In our univariate analyses, the cerebral NP score had a significant $(\mathrm{p}<0.0001)$ inverse association with MDI and survival, but after accounting for NFTs this association was not significant (Tables 4,5). Our measure of $A \beta$ accumulation included NPs only, rather than all forms of $A \beta$ plaques, which may underrepresent the contribution from $A \beta$. However, our multi-center cohort is one of the largest reported series of clinicallycharacterized patients with roughly equal numbers of PDD and DLB patients which allowed for comparisons across four levels of Braak/CERAD scores and a continuous measure of average cerebral NFT/NP scores. In contrast, previous studies had limited evaluations of NFT pathology by collapsing Braak stages for dichotomous comparisons ${ }^{8}$ or by examining categorical Braak NFT stages only ${ }^{20}$ in smaller samples with relative imbalances of $\mathrm{PDD} / \mathrm{DLB}^{20,21}$ or only non-demented PD or $\mathrm{PDD}^{8}$. Although in this study we focused on LBSD patients who developed dementia (PDD/DLB) and did not examine PD patients who came to autopsy prior to the onset of dementia (PDND), we have previously published a series of autopsy-confirmed PDND patients ${ }^{6}$ and found the majority of cases had a low level of tau pathology-35/44 (79\%) of cases had low Braak tau stages=0-II (i.e. tau pathology restricted to the hippocampus only) — which further reinforces our findings here. However, our results will need replication in an independent cohort to reconcile these discrepancies.

We also found age at death had an independent association with a longer MDI (Table 4), reflecting an overall longer disease course in patients with a long MDI. Thus, age-related factors may also contribute to these discrepancies, although in our multivariate regression correcting for age at death and sex, we found a significant independent association of NFT scores with a shorter MDI $(\mathrm{p}<0 \cdot 0001)$ and survival $(\mathrm{p}=0 \cdot 003)$. 
Although we did not include age at onset in our models because age at death more closely reflects the effects of aging on our measures of pathology found at autopsy, it may influence clinical phenotype in LBSD. A prospective autopsy study of idiopathic PD (Sydney Multicenter PD study) ${ }^{22}$ found three clinicopathological subtypes defined in-part by age at onset, where younger-onset PD patients were more likely to survive longer without dementia and have less co-morbid AD neuropathology compared to older-onset PD patients with a shorter survival and higher burden of secondary AD neuropathology. This study also found a small subgroup of six patients that had typical dopa-responsive PD and early dementia that met clinical criteria for DLB with a rapid disease course and high burdens of both SYN and AD neuropathology. These clinicopathological subgroups of PD patients are similar to our findings here and those previously published for PDND 6 . While important for providing novel insights into the progression of typical idiopathic PD, the Syndey Multicenter PD study $^{22}$ does not include all forms of clinical DLB, many of which do not have typical doparesponsive parkinsonism at onset. Indeed, in the current study a subset of individuals ( $n=18 / 98$ DLB, 18\%) never developed clinical motor parkinsonism during the course of their disease. Similar to the entire DLB cohort, the majority of these non-motor DLB individuals had intermediate to high levels of AD neuropathology $(16 / 18,89 \%)$ and neocortical LB stage $(15 / 18,83 \%)$.

Thus, this study and our current work provide complementary views of LBSD, and taken together, show a complex relationship between aging, SYN, and AD neuropathology. To resolve these discrepancies and more clearly define the clinicopathological subtypes of LBSD, future studies should quantify neuropathological burden parametrically, and use in vivo cerebrospinal fluid or imaging biomarkers of tau, A $\beta$, and SYN pathology in conjunction with detailed clinical data in patients who are prospectively followed to autopsy.

Nonetheless, our present data suggest that the consequences of AD neuropathology, and particularly NFT burden, are not solely an artefact of the aging process, but are instead central to the pathogenesis of the majority of LBSD patients. Indeed, we found that subjects with increasing AD neuropathology also had increasing levels of SYN pathology (Table 1) and a strong correlation between cerebral NFT, NP, and SYN scores (Figure 3), which suggests a synergistic effect of mixed AD and SYN neuropathology. We and others have previously shown associations of higher burden of AD neuropathology with more advanced SYN pathology in LBSD; ${ }^{6,8}$ cerebrospinal fluid markers of tau and SYN pathologies also appear to be highly correlated in $\mathrm{PD}^{23}$. These findings echo in vitro evidence for crossfibrillization of tau and SYN fibrils ${ }^{24}$ and conformational strains of pathological SYN that co-induce SYN and NFT pathology ${ }^{25}$. Future research is needed to further elucidate these potential mechanisms of co-accelerated pathology.

Other, less common co-morbid pathologic changes may be contributing to dementia in LBSD. Approximately 15-20\% of our cohort had co-morbid CVD or limbic TDP-43 pathology, with no clear association with AD neuropathology or with MDI and disease duration. The clinical significance of these and other less-common co-morbid pathologies (e.g. AGD, $\mathrm{HpScl}$ ) in our cohort is not certain and we cannot rule out a contribution of these pathologies to phenotypic diversity of LBSD on an individual patient level. Future work will need to assess these rare, co-morbid pathologies in a larger cohort. 
Another contributing factor to clinical heterogeneity in LBSD emerges from our genetic analyses. We found a higher frequency of both $G B A$ mutations and p.E326K polymorphism, which both confer risk of LBSD, ${ }^{12,26}$ in the low-level and no AD neuropathology groups (Table 2), and previously found a similar high rate of $G B A$ mutations in LBSD without comorbid AD in a cohort of patients with SYN pathology. ${ }^{12}$ We previously found $A P O E$ genotype to have an independent effect on the odds of dementia in $\mathrm{PD}^{6}$ and a higher frequency of the APOE \&4 allele in LBSD with SYN+AD and SYN-AD pathology both compared with controls ${ }^{11}$. Univariate analyses in this study found that the presence of $\geq 1$ $A P O E \& 4$ allele had an association with a shorter MDI and survival (Supplementary Figure 1), but these associations were not significant in the final multivariate models which included cerebral NFT and NP scores. Further study is needed to elucidate the mechanisms causing $A P O E$ and $G B A$ polymorphism to impact SYN pathology.

We did not find evidence for a definitive pathological substrate to support the categorical clinical distinction between PDD and DLB (Figure 1), despite the findings of higher cortical NFT, NP and SYN pathology in DLB compared with PDD (Supplemental Table 3); however, neuropathological assessment at autopsy may not accurately identify pathological differences that could potentially occur earlier in disease course. Interestingly, we found a consistent time interval from dementia onset to death of roughly 5 years across neuropathological (Table 3, Supplemental Table 1) and clinical groups (Supplemental Table 3). Previous studies in PD have shown a poor prognostic association of cognitive impairment in $\mathrm{PD}^{27,28}$ with a stereotypical decline to institutionalization and death 3-5 years after the onset of dementia ${ }^{29}$. Thus, the timing of dementia in LBSD is an important prognostic factor.

This study has several limitations inherent in a retrospective autopsy investigation. Indeed, some patients had missing clinical data; however, we had MDI and survival data for $>87 \%$ of the total cohort. All patients were referred to academic centers specialized in movement disorders and/or dementia so there may be a referral bias favoring atypical or more severe phenotypes. Replication of our findings in an independent prospective cohort is needed to further interpret the generalizability of results; however, we used a bootstrapped random patient selection procedure to reduce overfitting of our models. Finally, the neuropathological data were collected at autopsy from multiple centers using different staining procedures; however, we used standardized methods - used across >30 NIH/NIAfunded Alzheimer's centers in the National Alzheimer's disease Coordinating Center https:// www.alz.washington.edu/ - for merging multi-center data which provide reliability across participating institutions to reduce inter-lab variability, ${ }^{4,13}$ and the neuropathological methods and criteria used here were recently validated in a large multi-center study ${ }^{30}$.

Based on the strong associations of AD neuropathology (more specifically, NFT burden) with the time interval between motor symptom and dementia onset, and overall survival reported here, we suggest that future clinical diagnostic criteria for LBSD incorporate biomarkers for NFT and NP neuropathology for an individualized approach to diagnosis of underlying complex molecular pathology in LBSD and to identify patients at greatest risk for a more rapid decline. In addition, future clinical trials targeting SYN aggregation and propagation may benefit from stratifying analyses based on AD-related biomarker profiles 
and $A P O E$ genotype. Finally, our findings suggest that emerging therapies directed at the mitigation of pathological tau and $\mathrm{A} \beta$ amyloid could potentially slow the degenerative process and onset of cognitive difficulties in the majority of LBSD.

\section{Supplementary Material}

Refer to Web version on PubMed Central for supplementary material.

\section{Acknowledgments}

This study was supported by NIH grants: AG10124, P50 AG05136, AG006781, NS065070, AG028383, NS088341, AG08017, NS053488 and P50 NS062684. Drs. Tsuang and Zabetian are support by the Veterans Affairs Geriatric Research Education and Clinical Center, VA Puget Sound Health Care System. We would like to thank the patients and families who participated in this research, for whom this study would not be possible.

\section{References}

1. Emre M, Aarsland D, Brown R, et al. Clinical diagnostic criteria for dementia associated with Parkinson's disease. Movement disorders: official journal of the Movement Disorder Society. 2007; 22(12):1689-707. quiz 837. [PubMed: 17542011]

2. McKeith IG, Dickson DW, Lowe J, et al. Diagnosis and management of dementia with Lewy bodies: third report of the DLB Consortium. Neurology. 2005; 65(12):1863-72. [PubMed: 16237129]

3. Lippa CF, Duda JE, Grossman M, et al. DLB and PDD boundary issues: diagnosis, treatment, molecular pathology, and biomarkers. Neurology. 2007; 68(11):812-9. [PubMed: 17353469]

4. Montine TJ, Phelps CH, Beach TG, et al. National Institute on Aging-Alzheimer's Association guidelines for the neuropathologic assessment of Alzheimer's disease: a practical approach. Acta neuropathologica. 2012; 123(1):1-11. [PubMed: 22101365]

5. Aarsland D, Andersen K, Larsen JP, Lolk A, Kragh-Sorensen P. Prevalence and characteristics of dementia in Parkinson disease: an 8-year prospective study. Archives of neurology. 2003; 60(3): 387-92. [PubMed: 12633150]

6. Irwin DJ, White MT, Toledo JB, et al. Neuropathologic substrates of Parkinson disease dementia. Annals of neurology. 2012; 72(4):587-98. [PubMed: 23037886]

7. Ballard C, Ziabreva I, Perry R, et al. Differences in neuropathologic characteristics across the Lewy body dementia spectrum. Neurology. 2006; 67(11):1931-4. [PubMed: 17159096]

8. Compta Y, Parkkinen L, O'Sullivan SS, et al. Lewy- and Alzheimer-type pathologies in Parkinson's disease dementia: which is more important? Brain: a journal of neurology. 2011; 134(Pt 5):1493505. [PubMed: 21596773]

9. Irwin DJ, Lee VM, Trojanowski JQ. Parkinson's disease dementia: convergence of alpha-synuclein, tau and amyloid-beta pathologies. Nature reviews Neuroscience. 2013; 14(9):626-36. [PubMed: 23900411]

10. Gibb WR, Lees AJ. The relevance of the Lewy body to the pathogenesis of idiopathic Parkinson's disease. Journal of neurology, neurosurgery, and psychiatry. 1988; 51(6):745-52.

11. Tsuang D, Leverenz JB, Lopez OL, et al. APOE epsilon4 increases risk for dementia in pure synucleinopathies. JAMA neurology. 2013; 70(2):223-8. [PubMed: 23407718]

12. Tsuang D, Leverenz JB, Lopez OL, et al. GBA mutations increase risk for Lewy body disease with and without Alzheimer disease pathology. Neurology. 2012; 79(19):1944-50. [PubMed: 23035075]

13. Hyman BT, Phelps CH, Beach TG, et al. National Institute on Aging-Alzheimer's Association guidelines for the neuropathologic assessment of Alzheimer's disease. Alzheimer's \& dementia: the journal of the Alzheimer's Association. 2012; 8(1):1-13.

14. Nalls MA, Pankratz N, Lill CM, et al. Large-scale meta-analysis of genome-wide association data identifies six new risk loci for Parkinson's disease. Nature genetics. 2014; 46(9):989-93.

[PubMed: 25064009] 
15. Mata IF, Leverenz JB, Weintraub D, et al. GBA Variants are associated with a distinct pattern of cognitive deficits in Parkinson's disease. Movement disorders: official journal of the Movement Disorder Society. 2016; 31(1):95-102. [PubMed: 26296077]

16. Raftery AE. Bayesian Model Selection in Social Research. Sociological Methodology. 1995; 25:111-63.

17. Steyerberg EW, Harrell FE Jr, Borsboom GJ, Eijkemans MJ, Vergouwe Y, Habbema JD. Internal validation of predictive models: efficiency of some procedures for logistic regression analysis. Journal of clinical epidemiology. 2001; 54(8):774-81. [PubMed: 11470385]

18. Jellinger KA, Seppi K, Wenning GK, Poewe W. Impact of coexistent Alzheimer pathology on the natural history of Parkinson's disease. J Neural Transm (Vienna). 2002; 109(3):329-39. [PubMed: 11956955]

19. Sabbagh MN, Adler CH, Lahti TJ, et al. Parkinson disease with dementia: comparing patients with and without Alzheimer pathology. Alzheimer disease and associated disorders. 2009; 23(3):295-7. [PubMed: 19812474]

20. Ruffmann C, Calboli FC, Bravi I, et al. Cortical Lewy bodies and Abeta burden are associated with prevalence and timing of dementia in Lewy body diseases. Neuropathology and applied neurobiology. 2016 Aug; 42(5):436-50. [PubMed: 26527105]

21. Howlett DR, Whitfield D, Johnson M, et al. Regional Multiple Pathology Scores Are Associated with Cognitive Decline in Lewy Body Dementias. Brain pathology. 2015; 25(4):401-8. [PubMed: 25103200]

22. Halliday G, Hely M, Reid W, Morris J. The progression of pathology in longitudinally followed patients with Parkinson's disease. Acta neuropathologica. 2008; 115(4):409-15. [PubMed: 18231798]

23. Kang JH, Irwin DJ, Chen-Plotkin A, et al. Association of cerebrospinal fluid A $\beta 1-42$, t-tau, ptau181 and a-synuclein levels with clinical features of early drug naive Parkinson's disease patients. JAMA neurology. 2013; 70(10):1277-87. [PubMed: 23979011]

24. Lee VM, Giasson BI, Trojanowski JQ. More than just two peas in a pod: common amyloidogenic properties of tau and alpha-synuclein in neurodegenerative diseases. Trends Neurosci. 2004; 27(3): 129-34. [PubMed: 15036877]

25. Guo JL, Covell DJ, Daniels JP, et al. Distinct alpha-synuclein strains differentially promote tau inclusions in neurons. Cell. 2013; 154(1):103-17. [PubMed: 23827677]

26. Pankratz N, Beecham GW, DeStefano AL, et al. Meta-analysis of Parkinson's disease: identification of a novel locus, RIT2. Annals of neurology. 2012; 71(3):370-84. [PubMed: 22451204]

27. Litvan I, Goldman JG, Troster AI, et al. Diagnostic criteria for mild cognitive impairment in Parkinson's disease: Movement Disorder Society Task Force guidelines. Movement disorders: official journal of the Movement Disorder Society. 2012; 27(3):349-56. [PubMed: 22275317]

28. Pigott K, Rick J, Xie SX, et al. Longitudinal study of normal cognition in Parkinson disease. Neurology. 2015; 85(15):1276-82. [PubMed: 26362285]

29. Selikhova M, Williams DR, Kempster PA, Holton JL, Revesz T, Lees AJ. A clinico-pathological study of subtypes in Parkinson's disease. Brain: a journal of neurology. 2009; 132(Pt 11):2947-57. [PubMed: 19759203]

30. Montine TJ, Monsell SE, Beach TG, et al. Multisite assessment of NIA-AA guidelines for the neuropathologic evaluation of Alzheimer's disease. Alzheimer's \& dementia: the journal of the Alzheimer's Association. 2016 Feb; 12(2):164-9. 


\section{Research in Context}

\section{Evidence before this study}

We searched PubMed for original research articles from Jan 1, 2006, to April 25, 2016 using the terms "Lewy body dementia", "Parkinson's disease dementia" and "autopsy". We excluded non-autopsied biomarker studies and studies examining SYN pathology without clinical LBSD (e.g. co-morbid SYN pathology in clinical AD), since this was not the focus of our study. We found three recent studies which specifically addressed the neuropathological correlates of the MDI and survival across autopsy-confirmed clinical PDD and DLB patients. These studies found a higher deposition of cortical SYN and also AD-associated amyloid plaque (NP) pathology in DLB compared with PDD. Further, one study found both cortical amyloid plaques and SYN are associated with a shorter MDI and survival, while another used decline on a global measure of cognition to determine that a summation score of SYN, amyloid-plaque and tau NFT burden was the strongest correlate of cognitive decline in LBSD. The third study found similar higher burdens of cortical SYN and amyloid NP neuropathology in the short MDI groups (for both DLB and PDD patients with MDI 1-9.5 years) compared with PDD with a long MDI ( $>9.5$ years). One potential reason for these discrepancies between studies relates to sample size and relative frequencies of PDD and DLB patients, as each of these studies included $<55$ DLB patients. Another potential source of variability lies within the methodology for ascertainment of NFT burden, as two of the studies only used Braak staging as a measure of NFTs, which is largely based on topographical spread of pathology and not severity.

\section{Added value of this study}

To our knowledge, this is one of the largest multi-center cohorts of autopsy-confirmed LBSD patients with detailed clinical, genetic, and neuropathological data to provide a systematic examination of the neuroanatomical substrate of the heterogeneity in MDI and survival across LBSD. We included four stages of AD neuropathology in LBSD using modern neuropathological methods and criteria, and also examined continuous measures of $\mathrm{AD}$ and $\mathrm{SYN}$ pathology (i.e. average cerebral scores). We examined a range of other common co-morbid neuropathologic changes in LBSD, together with genetic risk polymorphisms for LBSD, to provide a comprehensive assessment of neuropathology in LBSD in our final multivariate models. Our outcomes of interest were continuous measures of MDI and survival, rather than the categorical clinical classification or nonspecific global measures of cognition used in the studies above. We found that increasing severity of the cortical burden of tau NFT pathology is associated with a shorter time course to develop dementia and death.

\section{Implications of all the available evidence}

The results of this study suggest that biomarkers of AD neuropathology may have important prognostic implications for clinical care and clinical trial design in LBSD. Current and future AD-targeted disease-modifying therapies may have the potential to attenuate cognitive symptoms in the majority of LBSD, as we found that increasing NFT severity was associated with decreasing MDI and survival. These observations will 
require replication in prospective cohorts of living patients using validated biomarkers of underlying AD neuropathology. 
A

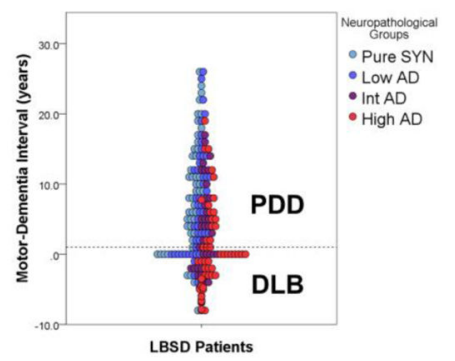

B

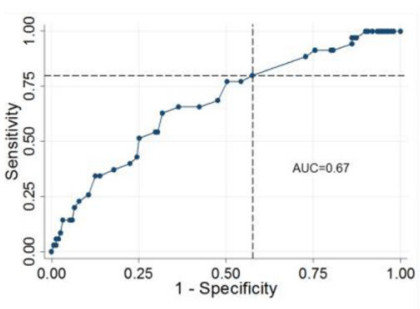

C

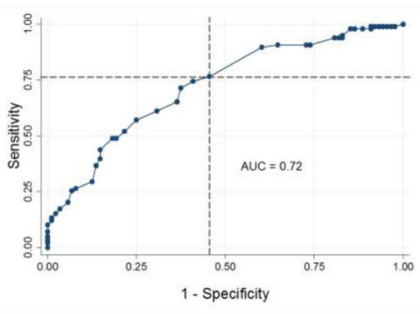

Figure 1. Diagnostic accuracy of the "one-year rule" to detect advanced SYN or AD neuropathology

A) Distribution of neuropathological AD groups among PDD and DLB clinical phenotypes in relation to the one year motor-dementia interval (MDI) rule (dashed line). B) ROC curve analysis testing the diagnostic accuracy of the MDI to distinguish neocortical stage of synucleinopathy. AUC $(0 \cdot 67, \mathrm{p}=0.05)$, sensitivity of $80 \%$, and specificity of $42 \%$ using the one-year rule (intersection of dashed lines). C) ROC curve analysis testing the diagnostic accuracy of the MDI to distinguish SYN+AD pathology. AUC $(0 \cdot 72, p=0 \cdot 0004)$, sensitivity of $76 \%$, and specificity of $55 \%$ using the one-year rule (intersection of dashed lines). 

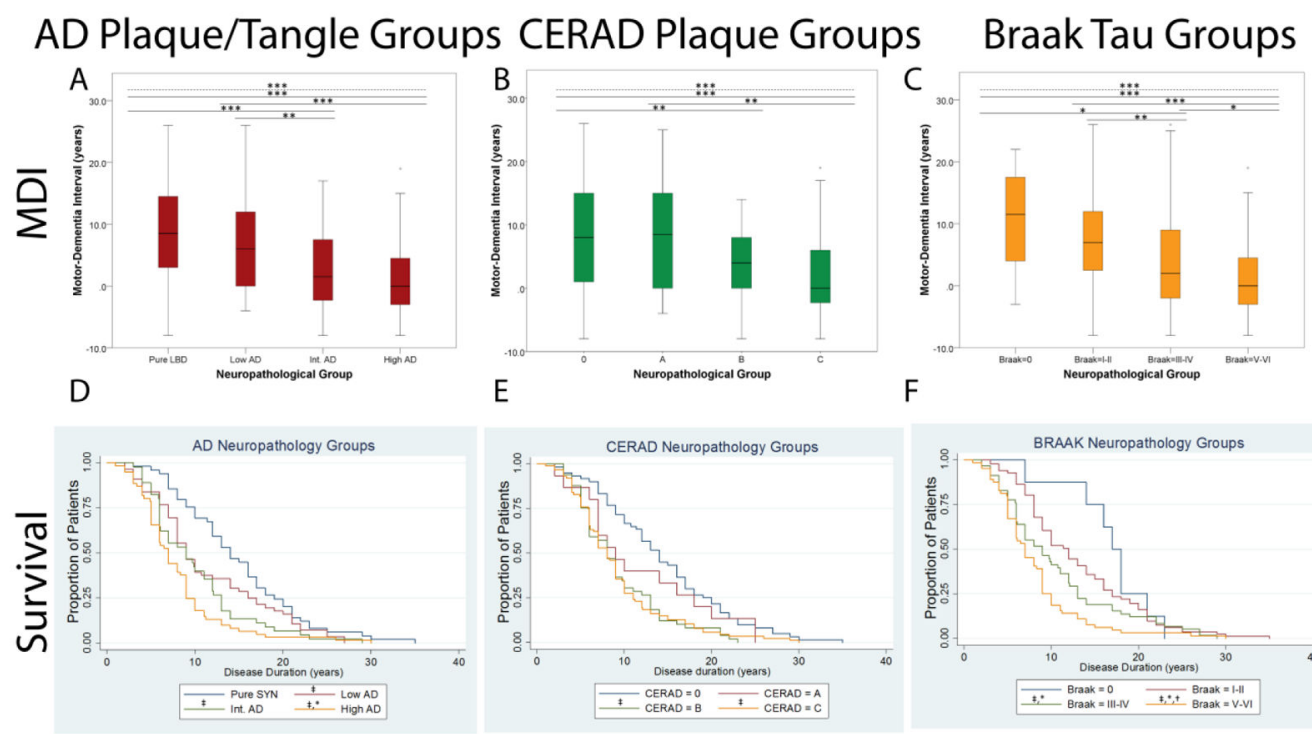

Figure 2. Comparison of motor-dementia interval and survival across Lewy body spectrum patients stratified by neuropathological group

Box-plots display the range of motor-dementia interval (MDI) for (A) total AD neuropathological change, (B) stages of senile plaque pathology, and (C) Braak stages of neurofibrillary tau. Dashed lines represent across-groups comparison (one-way ANOVA) and solid lines represent post-hoc individual group comparisons (independent t-tests) $* * *=\mathrm{p}<0 \cdot 001, * *=\mathrm{p}<0 \cdot 01, *=\mathrm{p} \unlhd 5 \cdot 05$. Kaplan-Meier curves depict the proportion of patients surviving at given time points observed for (D) total AD neuropathological change, $(\mathrm{E})$ stages of senile plaque pathology, and (F) Braak stages of neurofibrillary tau. Symbols above reference line denote: $\dagger \mathrm{p} \unlhd) .05$ compared to int.AD, * $\mathrm{p}<0.05$ compared to low AD/CA/BIII, $\ddagger \mathrm{p}<0.02$ compared to pure SYN/C0/B0 (independent sample t-tests). 
A

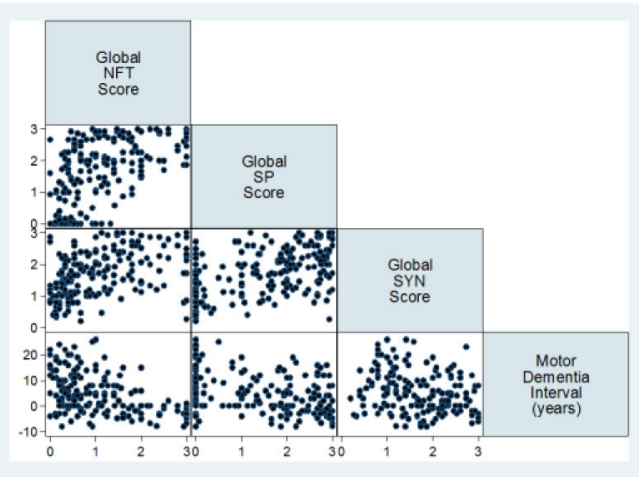

B

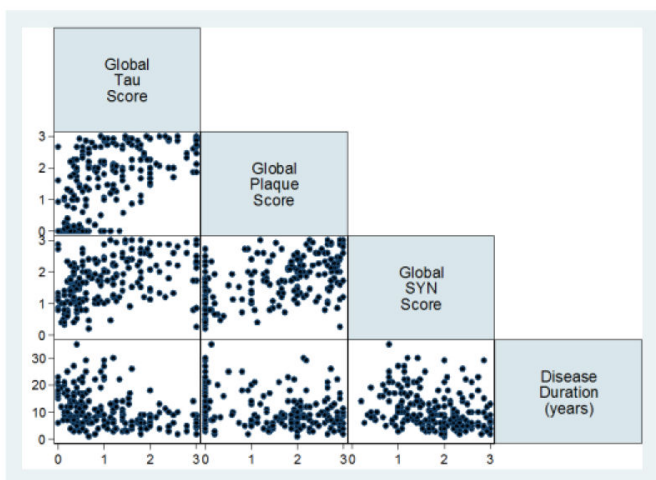

Figure 3. Comparison of cerebral average neuropathology scores with motor-dementia interval and survival in Lewy body spectrum disorders

Scatterplot matrices illustrate individual patient data correlations for each variable row/ column combination for (A) MDI and (B) survival. All cerebral pathologies scores are correlated with each other: Cerebral NFT: Cerebral NP (rho=0.6, p<0.0001), Cerebral NFT: Cerebral SYN (rho=0.5, p<0.0001), Cerebral NP: Cerebral SYN (rho=0.4, p<0.0001), inversely correlated with MDI: Cerebral NFT: MDI (rho $=-0 \cdot 4, \mathrm{p}<0 \cdot 0001)$, Cerebral NP: MDI (rho $=-0 \cdot 3, \mathrm{p}<0 \cdot 0001)$, Cerebral SYN: MDI (rho $=-0 \cdot 3, \mathrm{p}=0 \cdot 0001)$ and survival: Cerebral NFT: survival (rho $=-0 \cdot 4, \mathrm{p}<0 \cdot 0001)$, Cerebral NP $(\mathrm{rho}=-0 \cdot 3, \mathrm{p}<0 \cdot 0001)$ and Cerebral SYN: Survival (rho= $-0 \cdot 3, \mathrm{p}=0 \cdot 0001)$. 


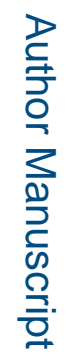

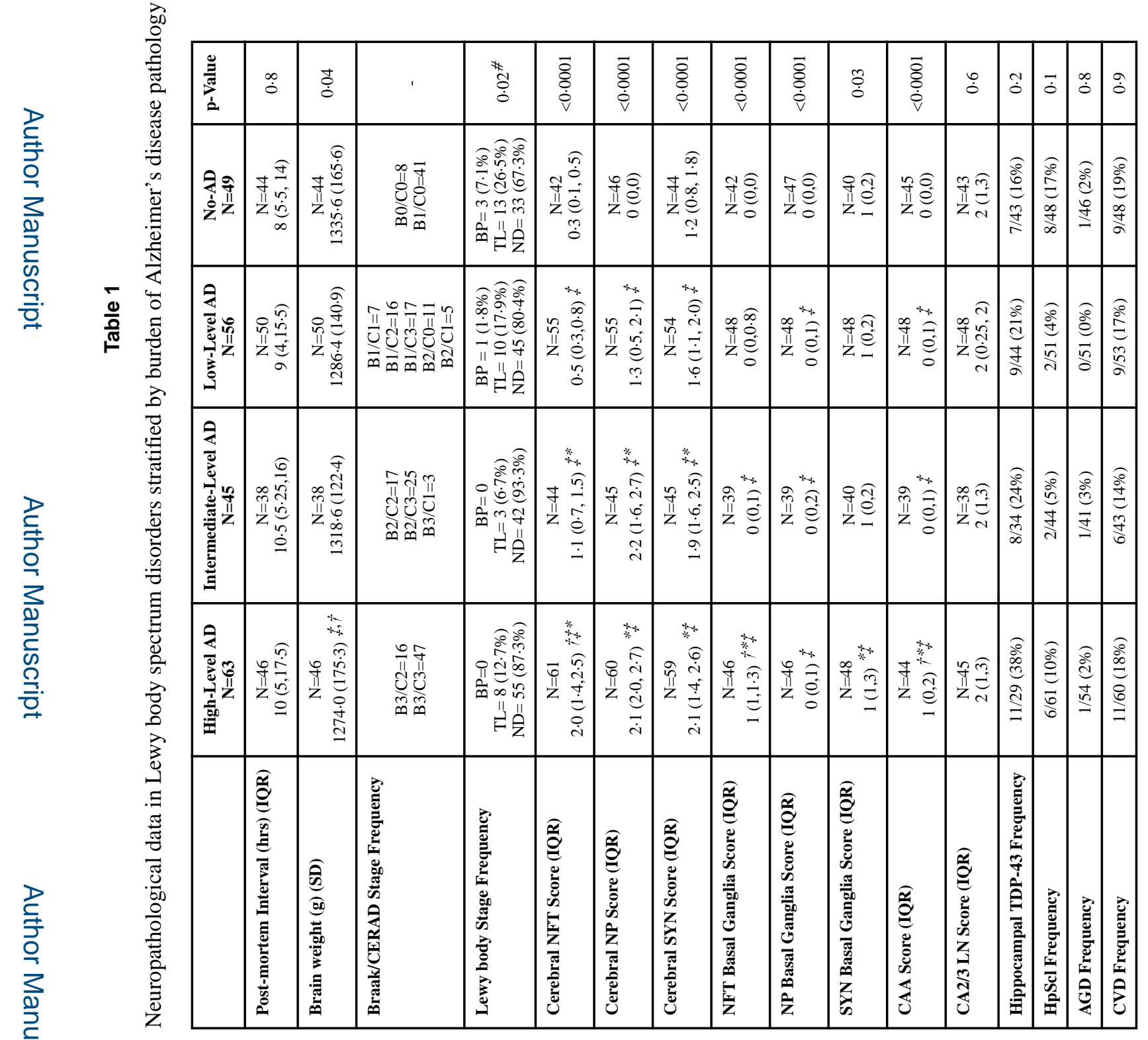

Lancet Neurol. Author manuscript; available in PMC 2017 January 01. 


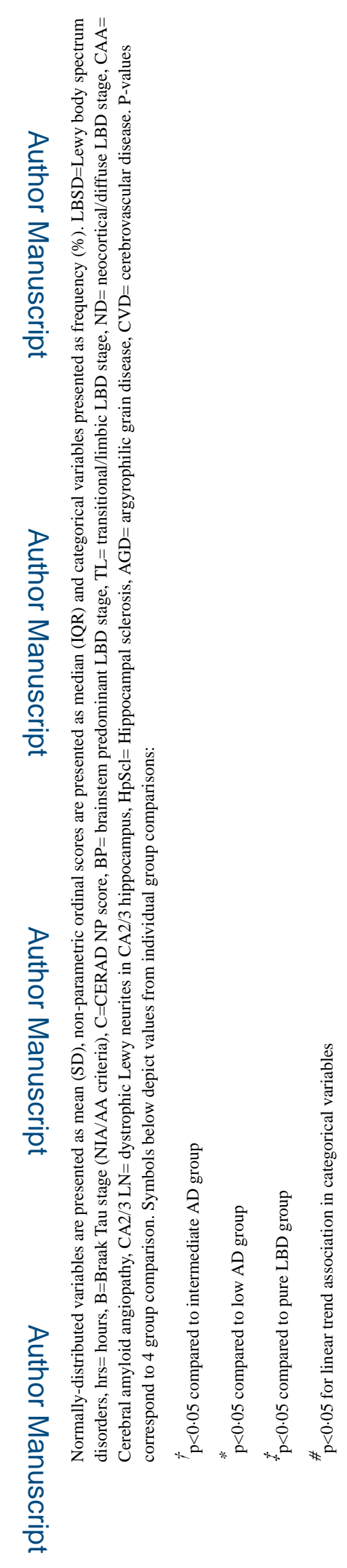




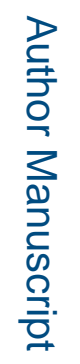

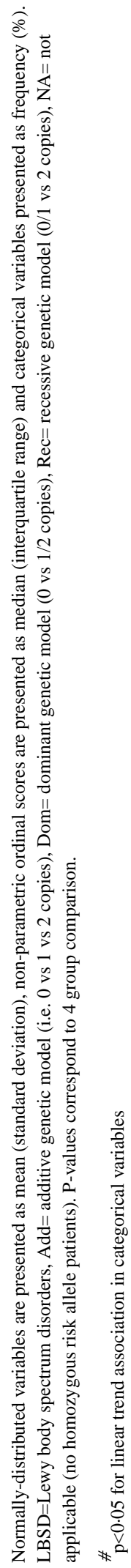

Lancet Neurol. Author manuscript; available in PMC 2017 January 01. 


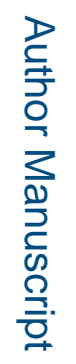

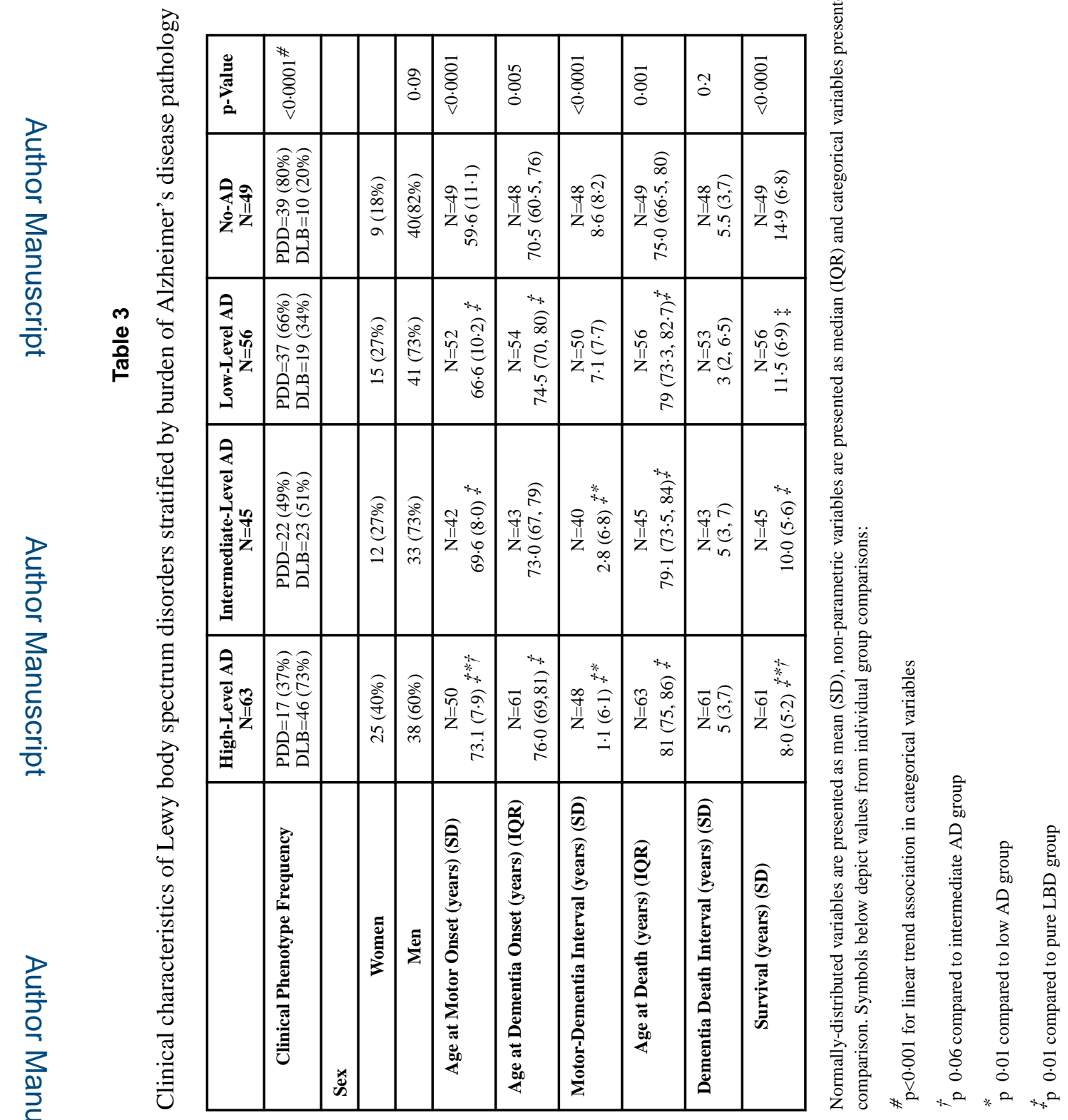

